\title{
Fluid dynamical phenomena in QGP and its recent experimental signatures
}

\author{
Laszlo P. Csernai* \\ University of Bergen, Norway \\ E-mail: laszlo.csernai@uib.no
}

Collective relativistic fluid dynamics is the dominant description of heavy ion reactions. With a Yang- Mills flux-tube initial state and a high-resolution (3+1)D particle-in-cell relativistic (PICR) hydrodynamics simulation, we calculate the shear flow, vorticity and Lambda polarization for different energies. The origin of polarization in high energy collisions is discussed, and we find linear impact parameter dependence of the global Lambda polarization. Furthermore, the global Lambda polarization in our model decreases very quickly with time in the low energy domain, and the decline curve fits well the recent results of Beam Energy Scan (BES) program launched by the STARCollaboration at the Relativistic Heavy Ion Collider (RHIC). The time evolution of polarization is also discussed.

Corfu Summer Institute 2018 "School and Workshops on Elementary Particle Physics and Gravity" (CORFU2018) 31 August - 28 September, 2018, Corfu, Greece

\footnotetext{
* Speaker.
} 


\section{Introduction}

Collective phenomena in high energy heavy ion reactions are adequately described by fluid dynamics. This is particularly the case when we are in the energy range where Quark Gluon Plasma (QGP) is formed and the number of degrees of freedom is of the order 100000 . It turned out that the QGP is rather behaving like a fluid and not like a dilute plasma, while the viscosity of the matter is minimal, especially at the phase transition domain of the Hadron Matter (HM) and QGP phase transition [1,2]. This observation is analogous to what was demonstrated at lower energies of 30 to $1000 \mathrm{MeV}$ per nucleon beam energies in the lab frame. At these energies, scaling violations to perfect fluid dynamics were indeed observed [3]. Due to these features in the last two decades we are using a high-resolution (3+1)D particle-in-cell relativistic (PICR) hydrodynamics model with extremely high resolution of several million "marker particles" in this computational model [4].

Collective flow effects show characteristic fluid dynamical features. Already in the early 1970s and 80 s, the observation of these led to the discovery of nuclear liquid-gas phase transition, and collective flow effects like bounce off, side splash, squeeze out, etc. Later on in the 1990s a new more regular characterisation spread: the directed, elliptical, triangular, ... (or $v_{1}, v_{2}, v_{3}$ ) flow. These collective flow patterns led to the accurate determination of the Equation of State (EoS) and transport properties of the HM and QGP. These collective phenomena required the study of peripheral collisions with different impact parameters. At the same time the use of oversimplified models, like the 1D Bjorken model, or spherical and 2D models became oversimplified, not realistic approaches.

Fluid dynamics describes a thermally equilibrated matter with a known EoS. Thus, the initial stage of the collision when the projectile and target matter reaches local equilibrium, and the final stage when the particles of the matter loose interaction with each other in the final expansion, require the use of additional dynamical models.

It is very important to have realistic initial models conserving the initial energy, momentum and angular momentum, as well as a realistic space-time configuration, which does not contradict to experimental observations. E.g. several models oversimplify the beam directed aspects of the initial state.

\section{The Initial State}

A realistic initial state model for the PICR computational model was worked out based on the Yang- Mills flux-tube initial state model, so that all conservation laws were satisfied [5, 5].

By now probably more than half if the experimental heavy ion research activity is invested into the study of collective effects or collective flow effects. The experimental methods are also continuously developing. To measure the collective flow effects one has to determine event by event $(\mathrm{EbE})$ the reaction plane (spanned by the beam axis $(z)$ and the impact parameter vector $b,(x))$. On the other hand, little attention is given to the longitudinal center of mass $(\mathrm{CM})$ of the participant system, which influences strongly the odd flow harmonics, $v_{1}, v_{3} \ldots$, and also the momentum dependence of the particle polarizations. Although, this was pointed out some time ago already [7], up to now, none of the experimental teams performs the EbE longitudinal CM 
momentum identification. Actually the inverse method can also be used. The global polarization should vanish EbE, for the $P_{x}$ and $P_{z}$ components, and the latter one can set the longitudinal $(z$ directed) $\mathrm{CM}$ momentum of the participants.

It is also important to separate the EbE random flow fluctuations from the global collective flow. This can also be done based on the given symmetries of the initial state [8]. The method introduced this way makes a reconstructible flow harmonics parametrization. In some cases, however, the experiments determine the reaction plane for each flow harmonics, $v_{1}, v_{2}, v_{3}$, separately, and in this way the flow pattern cannot be reconstructed from the flow harmonics, as each harmonics is given in a different frame!

The identification of the longitudinal CM of participants is important, especially as the softening of the directed flow or the anti-flow is a signal of QGP formation [9]. If the longitudinal CM of participants is fluctuating and it is not corrected EbE, then this directed flow signal is weakened or can even be eliminated [10].

We have seen that the collective flow is very sensitive for the initial state. Several results indicate that the Initial state is rather compact, and not extending to large pseudo-rapidities. This is in contrast to models, which assume longitudinal Bjorken or Gubser flow of large longitudinal extent, uniformly for all transverse points. In these types of approaches the angular momentum conservation can still be satisfied, but then the beam directed lengths of the projectile/target side will be at large positive/negative $z$-values, and there will not develop any shear-flow. Also, the above mentioned Yang-Mills model based initial state models will become excessively long in the $z$-direction.

To avoid this problem, a new initial state model was developed keeping the initial state compact, and maintaining significant shear flow [11]. This model is formulated in both Cartesian, $(t, x, y, z)$, and Milne, $(\tau, x, y, \eta)$, coordinates. In case of the Milne coordinates the origins of the centers of the different transverse, constant- $\tau$ hyperbolas, must have different origins, to have nonvanishing shear-flow.

\section{Shear-flow, Vorticity and polarization}

Stratified, shear flow, is well known and visible in nature, represented by turning waves on water or clouds in the sky. This phenomenon is known as Kelvin Helmholtz Instability (KHI). It is analyzed analytically and numerically, and was found that KHI may occur in heavy ion collisions. Only numerical methods with shear and with very small "numerical-viscosity" can reproduce this instability $[12,13]$. In heavy ion reactions with rapid explosive expansion, the development of $\mathrm{KHI}$ has limited time and leads an increased level of rotation. Compared to a 10 degree rotation as solid body before freeze-out, the KHI leads to more than 20 degree rotation. All the characteristic features of KHI development were reproduced by high resolution PICR model computations [12], while other numerical hydro models did not report to obtain this instability in realistic calculations.

Stratified shear flow, with compact initial state leads also to significant local vorticity, rot $v$ of the flow. In peripheral collisions this vorticity is an order of magnitude larger than the vorticity arising from random fluctuations! Furthermore the vorticity is arising from the initial angular momentum, pointing in the $-y$-direction, and thus the dominant vorticity direction is the same. This was first pointed out in ref. [14]. 
Interestingly and most importantly this vorticity is a very sensitive observable as it generates particle spin polarization as a consequence of spin-orbit interaction. Assuming final equipartition between spin and orbital angular momentum significant $\Lambda$ polarization was predicted [15]. The $\Lambda$ particle is special, as it is a "self analyzing" particle, so when it decays to a proton and a pion and the proton is emitted in the direction of the polarization (in the rest frame of the $\Lambda$. In case of relativistic heavy ions the final equipartition should take into account relativistic corrections.

The $\Lambda$ polarization in the participant centre-of-mass frame, as a function of its momentum, reads (in units $c=K=1$ ) [16]:

$$
\Pi_{\mu}(p)=\hbar \varepsilon_{\mu \rho \sigma \tau} \frac{p^{\tau}}{8 m} \frac{\int \mathrm{d} \Sigma_{\lambda} p^{\lambda} n_{F}\left(1-n_{F}\right) \partial^{\rho} \beta^{\sigma}}{\int \mathrm{d} \Sigma_{\lambda} p^{\lambda} n_{F}},
$$

where $\beta^{\mu}(x)=(1 / T(x)) u^{\mu}(x)$ is the inverse temperature four-vector field, $n_{F}$ is the Fermi-Jüttner distribution of the $\Lambda$, that is $1 /\left(e^{\beta(x) \cdot p-\xi(x)}+1\right)$, being $\xi(x)=\mu(x) / T(x)$ with $\mu$ the relevant $\Lambda$ chemical potential and $p$ its four-momentum. Because at the temperatures typical of freeze-out $\Lambda$ is quite dilute $\left(m_{\Lambda} \gg T\right)$, the Pauli blocking factor, $\left(1-n_{F}\right)$, can be neglected in Eq. (3.1).

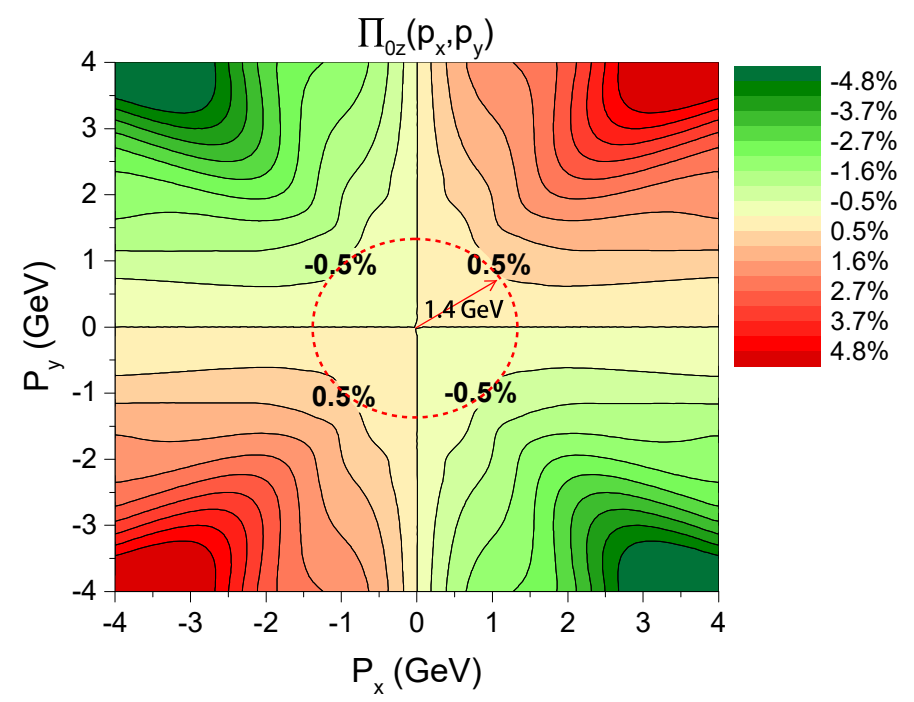

Figure 1: The transverse momentum distribution of longitudinal polarization, $\Pi_{0 z}$, with impact parameter ratio $b_{0}=0.68$ at the $200 \mathrm{GeV} \mathrm{Au}-\mathrm{Au}$ collisions. It has the correct sign distribution compared to the experimental data. The peak value for the $p_{T}=1.4 \mathrm{GeV}$ is about $0.5 \%$, the same with the global polarization at $b=0.5 \mathrm{fm} / \mathrm{c}$. This is in agreement with the experimental data, i.e. the peak value of longitudinal polarization at $p_{T}=1.4 \mathrm{GeV}$ has similar magnitude with the global polarization.

The spatial part of the polarization vector (3.1) gives rise to two terms:

$$
\begin{aligned}
& \Pi(p)=\frac{\hbar \varepsilon}{8 m} \frac{\int \mathrm{d} \Sigma_{\lambda} p^{\lambda} n_{F}(\nabla \times \beta)}{\int \mathrm{d} \Sigma_{\lambda} p^{\lambda} n_{F}} \\
& +\frac{\hbar \mathbf{p}}{8 m} \times \frac{\int \mathrm{d} \Sigma_{\lambda} p^{\lambda} n_{F}\left(\partial_{t} \beta+\nabla \beta^{0}\right)}{\int \mathrm{d} \Sigma_{\lambda} p^{\lambda} n_{F}} .
\end{aligned}
$$


These formulae apply to primary particles emitted from a locally equilibrated source. The first term corresponds to polarization due to the classical vorticity, while the second term contains the relativistic modifications.

The components, which have vanishing sum over all emission momenta, have typical symmetric structure. The $x, z$ plane is divided into four quadrants, with the signature starting from the $x$-axis counter clockwise, either (+-+-) or (-+-+). This is discussed extensively using the PICR fluid dynamical model $[17,18]$.

Interestingly the $\Pi_{1 z}(p)$ and the $\Pi_{2 z}(p)$ relativistic correction term have opposite signature. At low beam energies the primary classical term, $\Pi_{1 z}(p)$, dominates with (-+-+) signature, and the $\Pi_{2 z}(p)$ relativistic correction term (+-+-) is smaller. However, with increasing beam energy the relativistic term grows and the signature of the total polarization, $\Pi_{z}(p)=\Pi_{1 z}(p)+\Pi_{2 z}(p)$ changes to (+-+-) [19]. This last result is already in agreement with the experimentally observed signature.

These predictions were experimentally verified a few years after these predictions and became the most sensitive observables of collective motion in high energy heavy ion reactions. Due to the increased experimental sensitivity the non-dominant $x$ and $z$ directed polarizations and their momentum distributions are also measured. Furthermore, the difference between $\Lambda$ and anti- $\Lambda$ polarizations shows the same tendency at all measured beam energy, which led to different explanations, based on magnetic field effects arising from the rapid passing by of the spectators, as well as differences between baryon and anti-baryon interaction strength in the strong interaction [20]. In this work the baryon current was estimated in the simplest way by taking the $v_{1}$ flow component for the baryons. Recent experimental results on the other hand indicate that numerous baryon species and anti-baryon species show varying $v_{1}$ signatures. At the same time the dominant, $y$-directed polarization is rather stable and certainly not subject to sign changes.

\section{Summary}

We have seen that the features of the collective flow are decisive regarding many physical properties of the matter in both the HM and QGP phases, as well as their dynamics. Furthermore, the details of these new measurables are are highly sensitive, and can be decisive even for very small differences in theoretical models. This, certainly opens a new sensitive diagnostic possibility to de screen the until unknown details of matter and its interactions. The theoretical modeling can also be improved, and these sensitive experimental results give good guidance for these developments.

\section{Acknowledgement}

This work is supported by the Research Council of Norway under grant \# 255253/F50.

\section{References}

[1] L.P. Csernai, J.I. Kapusta, and L.D. McLerran, Phys. Rev. Lett. 97, (2006) 152303.

[2] P.K. Kovtun, D.T. Son and A.O. Starinets, Phys. Rev. Lett. 94, (2005) 111601.

[3] A. Bonasera and L.P. Csernai, Phys. Rev. Lett. 59, (1987) 630. 
[4] L.P. Csernai and D.D. Strottman, eds. Relativistic Heavy Ion Physics, Vol. 1, 2, World Scientific (1991).

[5] V.K. Magas, L.P. Csernai, and D.D. Strottman, Phys. Rev. C 64, (2001) 014901.

[6] V.K. Magas, L.P. Csernai, and D.D. Strottman, Nucl. Phys. A 712, (2002) 167.

[7] L.P. Csernai, G. Eyyubova and V.K. Magas, Phys. Rev. C 86, (2012) 024912, and ibid. 88, (2013) 019902(E).

[8] L.P. Csernai and H. Stöcker, J. Phys. G: Nucl. Part. Phys. 41 (2014) 124001.

[9] L.P. Csernai and D. Röhrich, Phys. Lett. B 458, (1999) 454.

[10] L.P. Csernai, V. K. Magas, H. Stöcker, and D.D. Strottman, Phys. Rev. C 84, (2011) 024914.

[11] V.K. Magas, J. Gordillo, D. Strottman, Y.L. Xie and L.P. Csernai, Phys. Rev. C 97 (2018) 064903.

[12] L.P. Csernai, D.D. Strottman and Cs. Anderlik, Phys. Rev. C 85, (2012) 054901.

[13] D.J. Wang, Z.Néda and L.P. Csernai, Phys. Rev. 87, (2013) 024908.

[14] L.P. Csernai, V.K. Magas and D.J. Wang, Phys. Rev. C 87, (2013) 034906.

[15] F. Becattini, L.P. Csernai and D.J. Wang, Phys. Rev. C 88, (2013) 034905.

[16] F. Becattini, V. Chandra, L. Del Zanna, and E. Grossi, Annals Phys. 338, (2013) 32.

[17] Yilong Xie, Robert C. Glastad, Laszlo P. Csernai, Phys. Rev. C 92, (2015) 064901.

[18] Y.L. Xie, M. Bleicher, H. Stöcker, D.J. Wang, L.P. Csernai, Phys. Rev. C 94, (2016) 054907.

[19] Y.L. Xie, et al., to be published

[20] L.P. Csernai, J.I. Kapusta and T. Welle, Phys. Rev. C 99, (2019) 021901(R). 